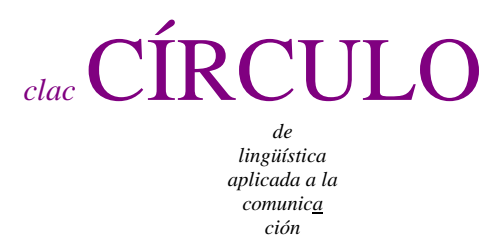

$58 / 2014$

\title{
ESTRATEGIAS DE ENCUADRE DISCURSIVO EN PERIODISMO POLÍTICO: ANÁLISIS DE UN CORPUS DE TITULARES
}

\author{
Beatriz Gallardo-Paúls y Salvador Enguix Oliver \\ Universitat de València
}

Beatriz Gallardo en uv es, Salvador Enguix en uv es

\section{Resumen}

En este trabajo combinamos la aplicación de conceptos de lingüística cognitiva y de teoría de la comunicación de masas para analizar un corpus de titulares de noticias de prensa escrita mediante un modelo de análisis del discurso centrado en cinco estrategias lingüísticas de encuadre: la estrategia intencional (tipo de acto de habla), léxica (usos marcados de la designación), predicativa (rentabilización de la iconicidad sintáctica en los actos expresivos), textual (uso de las superestructuras narrativa o argumentativa) e interactiva (dialogismo e intertextualidad). Se vincula el uso de estas estrategias a la mediatización periodística, que convierte el discurso de la comunicación política (institucional o partidista) en una enunciación incrustada, recursiva. Se comprueba que esta conversión obliga a un reajuste de estas estrategias respecto a cómo son utilizadas en el discurso de los partidos políticos.

Beatriz Gallardo-Paúls y Salvador Enguix Oliver. 2014.

Estrategias de encuadre discursivo en periodismo político: análisis de un corpus de titulares.

Círculo de Lingüística Aplicada a la Comunicación 58, 90-109.

http://www.ucm.es/info/circulo/no58/gallardo.pdf

http://revistas.ucm.es/index.php/CLAC

http://dx.doi.org/10.5209/rev_CLAC.2014.v58.45471

(C)2014 Beatriz Gallardo-Paúls y Salvador Enguix Oliver

Círculo de Lingüística Aplicada a la Comunicación (clac)

Universidad Complutense de Madrid. ISSN 1576-4737. http://www.ucm.es/info/circulo 
Palabras clave: Discurso político. Encuadre. Estrategias discursivas. Periodismo político.

\begin{abstract}
Discourse framing strategies in political journaliasm: Analysis of a corpus of headlines. In this paper the application of cognitive linguistics and mass communication theories are combined in order to analyze a corpus of news headlines, with a model of discourse analysis focused on five linguistic framing strategies. The strategies are: intentional (speech act), lexical (marked uses of denomination), predicative (application of syntactic iconicity in expressive acts), textual (use of narrative or argumentative superstructure) and interactive (dialogism and intertextuality). The use of these strategies in journalistic news is bound with mediatization proceses, that turn the discourse of political communication (institutional or partisan) in an embedded, recursive enunciation. It is found that this conversion requires a readjustment of these strategies with reference to how are they used in the discourse of political parties and politicians.
\end{abstract}

Key words: Political Discourse. Framing. Discursive strategies. Political Journalism.

Índice

Resumen 90

Abstract 91

1. Introducción 92

2. El corpus 93

3. Estrategias lingüísticas de encuadre 95

3.1. Estrategias de encuadre en el nivel enunciativo 97

3.2. La estrategia de encuadre en el nivel textual 102

4. Conclusiones 105

Bibliografía 106 


\section{Introducción}

Los estudios sobre discurso político y sobre medios de comunicación distinguen diversos modelos teóricos (Mazzoleni 1998, McNair 2000, Castells 2009) con los que dar cuenta de las relaciones entre los representantes políticos, los medios y la ciudadanía. En el modelo clásico de McNair, los medios ocupan un punto intermedio entre las instituciones políticas y los ciudadanos, y mantienen con ambos relaciones bidireccionales. En el modelo que Mazzoleni llama propagandístico-dialógico o de “comunicación mediatizada”, los procesos comunicativos entre las tres instancias son equiparables y son los puntos de intersección entre ellas los que sirven de escenario a la comunicación política, dándose la comunicación política mediatizada precisamente en el punto de central de confluencia de las tres. Por último, en el "modelo mediático de comunicación política” que surge a partir de la televisión, sólo este punto de triple intersección tiene importancia, de tal manera que los eventos comunicativos políticos que no acontecen en el escenario mediático pierden valor informativo. En palabras de Mazzoleni (1998: 28), “la actuación política pública se produce en la actualidad dentro del espacio mediático o depende en una medida significativa de la actuación de los medios”. Sería, pues, una política orientada a su mediatización (Kalb 1992; Casero 2008; Castells 2009).

En todo caso, con independencia de cuál de estos modelos teóricos se acepte, existen dos evidencias que resultan incuestionables: en primer lugar, que el discurso mediático constituye discurso político, y que por tanto participa de la construcción del marco interpretativo de dicho discurso: "mass media actively set the frames of reference that readers or viewers use to interpret and discuss public events”. (Scheufele 1999: 105). En segundo lugar, que simultáneamente supone una enunciación de segundo nivel, dependiente del primer nivel generado por los actores políticos institucionales. En este trabajo nos planteamos cómo esta situación de “enunciación incrustada” afecta y modifica la utilización de algunas estrategias lingüísticas de encuadre. 


\section{El corpus}

Aplicamos la teoría sobre las estrategias de encuadre discursivo a un corpus de titulares de prensa pertenecientes a las portadas de las ediciones nacionales de El País y El Mundo del año 2011. El corpus se diseñó siguiendo una de las metodologías más habituales en el análisis del contenido, la semana construida (Krippendorff, 1990). Para ello, se selecciona el primer día de la primera semana del período, el segundo de la segunda semana, y así sucesivamente, lo que permite obtener una muestra aleatoria que escapa a posibles distorsiones derivadas de períodos de tiempo más reducidos o continuados.

De este modo, el corpus final que analizamos en este trabajo lo integran los titulares de las noticias de portada de 46 ediciones impresas de los periódicos El País y El Mundo (en ambos casos la edición nacional), que pueden considerarse una muestra representativa del año en cuestión ${ }^{1}$. Esta selección de portadas nos proporcionó un total de 483 titulares de noticias, 410 de los cuales (un 84,9\%) fueron clasificados temáticamente como noticias de política nacional o internacional, incluyendo tanto la política en sentido estricto como noticias de política económica, u otras de relevancia para la interacción entre partidos e instituciones (por ejemplo, las noticias sobre corrupción); se excluyeron del análisis 73 titulares referidos a otros temas, como deportes, sociedad o cultura.

La selección de un corpus de titulares resulta especialmente ajustada para un estudio sobre el encuadre. Como es bien sabido, las investigaciones clásicas de los años 70 demostraron que los títulos (al igual que otros elementos paratextuales, como las ilustraciones) actúan como activadores del proceso mediante el cual se construye la comprensión textual. Desde planteamientos cognitivos, Jorques (2000: 97) ha señalado que el titular remite a una actividad de focalización atencional, cuya vinculación con la actividad de encuadre resulta evidente.

Aunque el titular como categoría textual específica de la prensa no es objeto de interés en este trabajo, cabe recordar algunos de los rasgos y funciones normalmente señalados por la bibliografía básica (Van Dijk 1980; Núñez Ladevéze 1991; De Fontcuberta

\footnotetext{
${ }^{1}$ Días 2, 10, 18 y 26 de enero; 3, 11, 19 y 27 de febrero; 7, 15, 23 y 31 de marzo; 8, 16 y 24 de abril; 2, 10, 18 y 26 de mayo; 3, 11, 19 y 27 de junio; 5, 13, 21 y 29 de julio; 6, 14, 22 y 30 de agosto; 7, 15 y 23 de septiembre; 1, 9, 17 y 25 de octubre; 2, 10, 18 y 26 de noviembre; y 4, 12, 20 y 28 de diciembre.
} 
1996). Se asume en general que los titulares de las noticias pueden funcionar como información, o como gancho apelativo. En el primer caso, los titulares informativos apuntan a un resumen de la noticia (macroestructura), y hay diversas propuestas que matizan esa informatividad diferenciando la función de resumen y la de anuncio, por ejemplo, o titulares informativos, interpretativos y explicativos. En el segundo caso, los titulares apelativos suponen algún tipo de reto a la comprensión que pretende estimular la curiosidad del lector; su utilización es mayor en los géneros de opinión, en los que la subjetividad enunciativa (la autoría) es más relevante.

$\mathrm{Al}$ hablar de la estrategia textual de encuadre retomaremos brevemente este aspecto y veremos que la mayoría de los titulares que integran el corpus cumplen una función semántica-informativa que adelanta el tema de que trata la noticia, y una función pragmático-cognitiva que orienta de manera más o menos marcada la interpretación y enfoque de la misma; aunque también interviene cierto matiz retórico de captatio, en la medida en que su redacción puede invitar o disuadir de la lectura completa de la noticia, se trata de un aspecto secundario (a diferencia, por ejemplo, de los titulares de los editoriales). Muchas veces los titulares de noticias de los dos diarios analizados reflejan estructuras muy similares, que parecen reproducir miméticamente textos previos procedentes de las agencias de prensa o los gabinetes de comunicación:

[Ej. 01] Cascos se va del PP con un duro ataque a Rajoy [EM, 02.01.2011]

[Ej. 02] Cascos abandona el PP con duras acusaciones contra Rajoy [EP, 02.01.2011]

La selección de titulares de portada obedece a su representatividad, y es práctica habitual en el análisis del contenido; los equipos de dirección y los consejos editoriales de los diarios son conscientes de la importancia de esta selección informativa por sí misma y de cara al lector y la opinión pública, pero también como elemento que será divulgado y amplificado en los sumarios de los medios audiovisuales (Casero y López 2012; [referencia eliminada]). En otros términos podría afirmarse que la inclusión de cierta noticia en portada constituye ya una actividad de encuadre, al igual que lo es su focalización como noticia de apertura; las teorías de fijación de la agenda, priorización y encuadre (agenda-setting, priming, framing) son, de hecho, teorías interrelacionadas, que la bibliografía suele utilizar de manera superpuesta (a veces propiciando cierta interpretación difusa, Scheufele 1999; Entman 2009, 2010; Castells 2009). 


\section{Estrategias lingüísticas de encuadre}

Como es sabido, existe una notable tradición de análisis de los procesos de encuadre (framing) realizados en las noticias y en otros géneros periodísticos; aunque el concepto se desarrolla en las ciencias sociales desde la década de los 50 y 70 (Bateson, Goffman), su consolidación definitiva en el ámbito de la comunicación de masas suele atribuirse a la propuesta de Entman (1991, 1993, 2009). Por lo general, este concepto de encuadre ${ }^{2}$ atiende a aspectos específicamente semánticos de la enunciación, que se identifican a partir de factores como las repeticiones léxicas, las conceptualizaciones metafóricas ${ }^{3}$, la selección temática, o las expresiones valorativas ${ }^{4}$. Nuestro análisis, no obstante, se plantea un enfoque pragmático más amplio que junto a los aspectos enunciativos (donde, efectivamente, los procesos de articulación semántica son fundamentales), considera también estrategias textuales e interactivas. Se trata de estrategias que pueden considerarse implícitas, puesto que orientan la interpretación de manera indirecta, como elementos añadidos al texto explícito; desde el punto de vista pragmático pueden considerarse huellas formales de la enunciación en el sentido de Benveniste.

No consideraremos aquí, sin embargo, otras estrategias no lingüísticas que son más asimilables al paratexto icónico; por ejemplo, Entman (1991: 10) señala que uno de los elementos del encuadre es el espacio y la cantidad de información de cada noticia (también si hay o no imágenes, el tamaño del titular, el ser o no noticia de apertura, su longitud, etc.), pues estos elementos correlacionan con la importancia informativa (la importancia política) que se les adjudica. Cabría añadir que, además, existe un elemento inicial de encuadre informativo constituido por la propia cabecera de cada diario; de

\footnotetext{
${ }^{2}$ Entman (2009: 5) define el encuadre como "selecting and highlighting some facets of events or issues, and making connections among them so as to promote a particular interpretation, evaluation, and/or solution".

${ }^{3}$ La metáfora de las relaciones familiares (el "padre estricto" vs. el "progenitor protector") ha sido bien analizada por Lakoff (2004) para describir los encuadres metafóricos de los partidos estadounidenses demócrata y republicano; Entman (2009, 2010) ha descrito también la metáfora del "diablo” (y del “terrorismo diabólico”) utilizada por Bush para referirse a Bin Laden y Al Qaeda tras el 11S.

${ }^{4}$ Por ejemplo, en el análisis del “encuadre sesgado” que realiza a propósito de la campaña presidencial de 2008, Entman (2010: 396) codifica (como positivos o negativos) los enunciados valorativos sobre los candidatos y los partidos a partir de tres dimensiones: 1) personalidad y aptitudes, 2) habilidades de campaña y posición política, y 3) planteamientos políticos.
} 
hecho, podría decirse que para el lector prototípico la lectura de un titular se orienta interpretativamente desde el momento en que sabe si pertenece a El Mundo o a El País, pues esta asignación se convierte en activadora de inferencias e implícitos culturales.

Como hemos adelantado, el análisis repasa los tres niveles pragmáticos fundamentales, identificando ciertas estrategias que se superponen al contenido del texto:

1. Estrategias de encuadre enunciativo, donde se diferencia una dimensión intencional (correspondiente al acto de habla ilocutivo), una dimensión léxica (utilización de connotaciones, usos equívocos o ambiguos mediante la designación, activación de relaciones léxicas), y una dimensión predicativa (recursos sintácticos que contribuyen al encuadre).

2. Estrategias de encuadre textual, es decir, la identificación de la estructura textual básica a la que remite el texto en cuestión, narrativa o argumentativa.

3. Estrategias de encuadre interactivo, es decir, los mecanismos lingüísticos de atribución discursiva, que pueden ser interpretados como huellas de subjetividad y dialogismo.

Estudios previos sobre estas estrategias en el discurso de los partidos políticos (Gallardo 2013, 2014) muestran que los argumentarios de partido utilizan sistemáticamente la estrategia léxica (relativizando el significado de las palabras clave del campo semántico de la política), privilegian una estrategia intencional de ilocutividad expresiva (que se dedica sobre todo a hablar valorativamente de los propios políticos con actos de habla de ataque y autoalabanza, descuidando los temas del bien común), y rentabilizan la iconicidad sintáctica (presentando como sujeto a los destinatarios de tales actos de habla de ataque o autoelogio). En el nivel textual, el discurso de los argumentarios políticos adopta formatos aparentemente argumentativos para transmitir contenidos narrativos. Por último, en el plano interactivo explotan la estrategia dialógica para vincular siempre cada mensaje con su emisor, especialmente para rentabilizar el contraste partidista. Revisamos a continuación cómo estas mismas estrategias, que se superponen al contenido referencial de cada texto, se despliegan en los titulares de noticias de prensa escrita. 


\subsection{Estrategias de encuadre en el nivel enunciativo}

En el encuadre lingüístico enunciativo, vinculado al acto de habla del sujeto emisor, confluyen tres tipos de mecanismos o estrategias: intencional (ilocutividad), léxica (designación) y predicativa (sintaxis).

La estrategia intencional de encuadre corresponde a la intención comunicativa básica del emisor, es decir, la ilocutividad de su acto de habla ${ }^{5}$. Lo esperable en el discurso periodístico es, obviamente, un predominio de actos representativos asociados a la actividad de informar, pero también corresponde a esta estrategia transmitir la ilocutividad expresiva de adhesión y discrepancia, es decir, de alabanza y ataque a una u otra opción política.

Los estudios comparativos centrados en la praxis profesional y la visión que tienen los propios periodistas políticos de su trabajo han señalado ${ }^{6}$ que los periodistas españoles, frente a sus colegas del norte y centro de Europa, tienden a mantener unas relaciones más simbióticas que competitivas con los políticos parlamentarios, y que su actitud es en general más partidista y parcial; el periodismo político de los medios españoles sería según estos trabajos, un periodismo más idealista que pragmático, y la prensa tendría una orientación partidista relativamente definida (Van Dalen 2010; Berganza, Van Dalen y Chamorro 2010; Hanitzsch et al. 2011; Van Dalen, de Vreese y Albaek 2012; Hopman et al. 2012). Desde el punto de vista discursivo, cabe plantearse si esta actitud supone redactar los textos informativos con una aceptación de los marcos lingüísticocognitivos propuestos por los emisores políticos, manteniendo en general las perspectivas de interpretación que emanan desde las distintas esferas de poder y, por

\footnotetext{
5 Asumimos la tipología clásica de Searle sobre actos ilocutivos: representativos, compromisorios, directivos, expresivos y declarativos. Todos ellos están presentes en el discurso político, y se distribuyen de manera predominante en los diferentes eventos y situaciones comunicativas en que este tiene lugar. Por ejemplo, la retórica electoral se mueve entre los polos del acto directivo (“votadme”) y compromisorio (“esta será mi política”).

6 Estos estudios comparados se basan generalmente en encuestas a profesionales. Por ejemplo, Van Dalen, de Vreese y Albaek (2012) comparan las actitudes profesionales de periodistas políticos británicos, españoles, alemanes y daneses, a partir de preguntas que reflejan tres dimensiones: 1) el enfoque idealista o pragmático de las noticias políticas, es decir, con una visión de mayor implicación o más distanciada y cínica; 2) el grado de compromiso partidista del medio en que trabajan: imparcial vs. partidista; y 3) la orientación de la audiencia hacia la información o hacia el entretenimiento, es decir, la concepción del público como ciudadano o como consumidor.
} 
extensión, las mismas estrategias lingüísticas que les dan soporte. De ser así, la estrategia intencional supondría reproducir de forma especular el encuadre de ataque o alabanza transmitido por el discurso de los representantes políticos, en detrimento de la ilocutividad representativa, de expresividad neutra, que parece la más ajustada a la actividad informativa. La línea de investigación que se desarrolla a partir de teorías como "la espiral de cinismo" o la metáfora de la "carrera de caballos" en el periodismo electoral (Capella y Jamieson 1996; Van Zoonen 2005), reflejarían parcialmente esta perpetuación de los marcos intencionales, si bien la posición partidista en las noticias periodísticas puede vehicularse mediante otros mecanismos, como la selección de la agenda temática ${ }^{7}$ o la dosificación informativa.

Como ya hemos mencionado, en términos generales puede afirmarse que el discurso político de los partidos y sus representantes muestra un claro predominio de la intencionalidad expresiva frente a la representativa ${ }^{8}$. Es decir, gran parte del discurso político se dedica a hablar valorativamente de sus propios protagonistas, y relega los temas del bien común (de la política) a un segundo término (Van Zoonen 2005; Timoteo 2007; Castells 2009).

Contrariamente, el análisis del corpus de titulares de prensa confirma el predominio de los enunciados neutros (un $81 \%$ de los titulares), que transmiten hechos con una ilocutividad representativa que se aproxima al ideal de la objetividad periodística; no hay, por tanto, un mantenimiento de la ilocutividad expresiva privilegiada en los mensajes de los representantes políticos. Esta diferencia se justifica pragmáticamente

\footnotetext{
${ }^{7}$ Por ejemplo, aun tratándose de un corpus de muestra aleatoria, resulta significativo que mientras en los días seleccionados El Mundo incluyó en portada 6 noticias sobre el llamado “caso Faisán”, las portadas de los mismos días de El País incluyen solo una noticia sobre el mismo tema. (Titulares EM: 26 de enero: "Los policías del chivatazo no quieren revelar al juez la identidad del confidente"; 3 de febrero: "González Pons: 'Si el chivatazo se dio desde Interior, Rubalcaba estaría inhabilitado para la vida política'”; 11 de febrero: "Ruz investiga si el chivatazo fue parte de la negociación con ETA"; 19 de febrero: "El fiscal contradice la versión de Rubalcaba sobre el Faisán”; 16 de abril: "Rubalcaba da su apoyo a los ataques al Informe Final del chivatazo"; 23 de septiembre: "Interior asciende al policía acusado del chivatazo a ETA". Titular EP: 23 de septiembre: "Los imputados por el "Faisán" piden investigar al policía que les acusó”).

${ }^{8}$ El análisis ya citado de argumentarios del PP y el PSOE redactados entre 2008 y 2013 revela que un $58,5 \%$ de los enunciados se dedican a atacar o elogiar a los representantes y partidos políticos, mientras un $41,5 \%$ se dedica a hablar sobre temas de la realidad política (Gallardo 2014).
} 
porque, aunque se refieran a lo mismo, ambos discursos suponen niveles de enunciación diferentes. Como veremos al hablar de la intertextualidad, es habitual que los mensajes del discurso periodístico transformen en noticia las enunciaciones del discurso político de primer nivel, convirtiendo la enunciación del protagonista político en una enunciación incrustada que puede ser referida sin implicación subjetiva; convirtiéndola, por tanto, en enunciado, en tema. Como se ha dicho, la propia cabecera de cada periódico ya proporciona un encuadre inicial a la lectura de cada titular, de tal manera que pese a que la emisión adopte una ilocutividad neutra, representativa, secundariamente puede activarse la intención de alabanza o ataque según el rotativo que contiene la noticia en cuestión ${ }^{9}$. No obstante, en el corpus existen también titulares claramente alineados con una posición política o partidista: junto a los titulares representativos vemos que un $18 \%$ del total de enunciados son actos de ataque, y un $1,7 \%$ actos de alabanza o apoyo evidente a un líder o partido político ${ }^{10}$.

El análisis de la estrategia intencional, por tanto, es doblemente interesante: en primer lugar, porque permite comprobar que esa parcialidad o enfoque sesgado que la bibliografía propone a veces para la prensa española no se confirma en la dimensión ilocutiva de los titulares de noticias y, por tanto, debe ser sustentada en otros mecanismos; los datos no muestran que ese alineamiento partidista se plasme lingüísticamente con los mismos actos de habla expresivos que usan los políticos, sino que evidencian el esperable predominio de los actos representativos, referenciales. En segundo lugar, porque aleja el discurso periodístico del discurso de la comunicación política, desarrollando la función de mediatización discursiva que le es propia.

En la estrategia léxica el emisor del discurso político realiza el acto de designación apoyándose en un uso semántico ambiguo, impreciso o sesgado, que explota las relaciones léxicas y la connotación, activando esferas de significación que exceden o solapan la dimensión informativa estricta del mensaje; obviamente, esta elección de las

\footnotetext{
9 Según las teorías que convierten la prensa en “periodismo de convalidación” (Timoteo 2007: 367), cabría afirmar que en realidad esa actitud de ataque o alabanza partidista es previa al propio acto de lectura.

${ }^{10}$ Este es un punto en el que los dos periódicos muestran diferencias; El País construye con ilocutividad representativa un 92,6\% de sus enunciados, mientras en El Mundo esta cifra es del 69,6\%.
} 
palabras se pone al servicio de la estrategia intencional. Esta estrategia de encuadre afecta al nivel léxico de la palabra concreta, frente a la estrategia semántica general que permite encuadrar cada noticia mediante la selección informativa que ordena focos y presuposiciones, como ilustra el siguiente par de titulares relativo a la misma noticia (la estrategia léxica aparece en el uso de “minipacto”):

[Ej. 03] El rebrote del paro estropea el minipacto de La Moncloa [EM, 03.02.2011]

[Ej. 04] Zapatero resalta el pacto social como marca de confianza en España [EP, 03.02.2011]

Tal y como ha señalado recurrentemente la bibliografía, el uso léxico marcado, que fuerza al máximo los límites de la prevaricación (entendida como rasgo semiótico), define la esencia del uso discursivo de los representantes e instituciones políticas (Edelman 1985; Ricoeur 1987; Wodak 1989; [referencia eliminada]). Por ejemplo, se utilizan como sinónimos términos que no lo son (usar "reforma" como equivalente de “recorte presupuestario”), se recurre a la metáfora o la ironía para activar connotaciones (“Mariano Rajoy encontró ayer la piedra filosofal de la austeridad y la contención en el gasto. Hoy no sabe cómo usarla” [Argumentario PSOE, 02.06.2011]), se explotan los usos equívocos y difusos con intención eufemística (llamar "movilidad exterior" al éxodo de jóvenes sin trabajo que otros designan como "exilio económico"; nombrar cierta subida del impuesto sobre la renta como "recargo temporal de la solidaridad"; denominar "atentados” las manifestaciones de protesta ciudadana o equipararlas al nazismo), se utilizan coloquialismos que enmascaran la hipérbole (“El Gobierno presenta a Europa las medidas para salir del agujero en que nos metieron las políticas socialistas” [Argumentario PP, 27.04.2012]) o directamente se inventan usos léxicos cuyo significado a duras penas puede inferirse cotextualmente ("La tasa de paro flexiona en 2014 (hasta el 26,7\% de la población activa), para situarse por debajo del 25\% en 2015”, Nota de prensa de Moncloa, 26.04.2013).

Todos estos mecanismos léxicos responden al concepto de articulación (Howarth 1995), según el cual el significado de los términos del discurso político depende de un término previo que les da sentido; la noción es similar a lo que en lingüística cognitiva se denomina habitualmente encuadre (frame) (Fillmore 1976; Lakoff 1992, 2004), 
aunque la articulación suele poner en juego dos términos que se condicionan entre sí ${ }^{11}$. En definitiva puede afirmarse que el discurso político estricto (primer nivel de enunciación), generado por los representantes de instituciones y partidos, se caracteriza por la actualización constante de procesos de articulación y encuadre, a partir de los cuales el significado de ciertos términos se relativiza y modifica, tanto para incorporar connotaciones y asociaciones como para ignorar parte de la carga semántica apropiada; en otras palabras, para orientar ideológicamente la interpretación.

Por su parte, el discurso político periodístico no muestra el mismo despliegue de la estrategia léxica que puede observarse en el discurso de partidos y representantes según lo acabamos de describir. Al igual que ocurre en el ámbito intencional de los actos de habla, en la medida en que la enunciación periodística constituye un proceso de mediatización, de puente entre el político y el ciudadano, parece esperable que, efectivamente, el uso léxico sea más ajustado a la semántica de la lengua y no recurra a estrategias tan evidentes de articulación. Y así es. El corpus muestra escasa utilización de este mecanismo, y los usos léxicos que llaman la atención responden en general a la introducción de coloquialismos o matices valorativos que son relevantes para la estrategia intencional, pero solo ocasionalmente suponen un uso distorsionado tan evidente como en el primer nivel del discurso político.

Un recurso de la estrategia léxica consiste en la explotación de presuposiciones, especialmente la presuposición existencial, que permite introducir en la esfera de lo real contenidos cuya veracidad se da por sentada. El siguiente titular selecciona un término marcado peyorativamente (“chivatazo”, frente a otras posibilidades más neutras, como “filtración”), y lo introduce con artículo reconocedor, lo que activa su condición existencial como algo verdadero (y no “presunto”, por mencionar un adjetivo del que la prensa suele abusar en estos casos):

[Ej. 05] Los policías del chivatazo no quieren revelar al juez la identidad del confidente [EM, 26.01.2011]

En otras ocasiones, como hemos mencionado, el uso léxico valorativo (“apropiarse de”) permite también activar la estrategia intencional expresiva:

\footnotetext{
${ }^{11}$ La cabecera de cada diario actúa como base para la articulación, mientras en el discurso de los representantes políticos la articulación surge del polo ideológico "nosotros/ellos”.
} 
[Ej. 06] La Iglesia se apropia de miles de templos, casas, parcelas o viñedos [EP, 11.07.2011]

De hecho, a medio camino entre la ilocutividad estrictamente representativa, que refiere un estado de cosas, y la ilocutividad expresiva que se alinea ideológicamente con una opción política determinada, existe un punto intermedio que se corresponde con la naturaleza intermedia de la enunciación periodística: el de los titulares interpretativos. Estos titulares no pueden catalogarse como claramente orientados a favor o en contra del tema que tratan, pero sí introducen cierto matiz que dirige la interpretación y propone el encuadre valorativo.

Por último, sin abandonar el ámbito enunciativo, la estrategia predicativa rentabiliza la iconicidad sintáctica colocando como sujeto de las oraciones al protagonista de la estrategia intencional; obsérvese la diferencia entre los dos titulares:

[Ej. 07] Rajoy sacrifica a Camps [EM, 21.07.2011]

[Ej. 08] Camps se rinde [EP, 21.07.2011]

Su eficacia es mucho mayor en los enunciados de alabanza o ataque, es decir, de ilocutividad expresiva. En el análisis de los titulares encontramos que esta estrategia es utilizada con frecuencia, lo que consigue reforzar la intencionalidad de elogio o ataque partidista (se aplica en el 58\% de los titulares de intencionalidad expresiva). Por ejemplo, en el siguiente enunciado, el ataque al PSOE no recurre a la estrategia predicativa:

[Ej. 09] Un falso prejubilado donó al PSOE la sede de Camas [EM, 15.03.2011]

Mientras en los siguientes casos se complementan ambas estrategias, a las que se suma el efecto de la estrategia léxica que supone la elección de términos como “atrincherarse”, “mentira”, “jalear”, o "al filo del banquillo”:

[Ej. 10] Rubalcaba se atrinchera en la mentira de su nota oficial [EM, 07.03.2011]

[Ej. 11] Rajoy jalea ante miles de fieles a un Camps al filo del banquillo [EP, 18.05.2011]

3.2. La estrategia de encuadre en el nivel textual

El encuadre del discurso político depende también del tipo de estructura textual que activa cada texto; las dos modalidades básicas, narración y argumentación, arrastran una 
serie de asociaciones cognitivas cuya movilización resulta muy importante en el discurso de los actantes políticos, pero que en el periodismo político está condicionada por cada género textual concreto. Así, la narración remite a la verosimilitud, la metáfora, la ficción, el relato o las realidades psíquicas, en oposición a la verdad, la metonimia, la lógica, la demostración o las realidades objetivas que se asocian a la argumentación (Bruner 1986; Fisher 1984).

Obviamente, los titulares de las noticias son candidatos naturales para la activación de modelos textuales narrativos por la propia dimensión narrativa del género noticia ${ }^{12}$. De ahí el predominio de estructuras proposicionales (solo 3 titulares no evocan una estructura narrativa), que contrasta con los titulares de otros géneros periodísticos como el editorial, donde es mucho más frecuente encontrar textos que activan estructuras claramente argumentativas, y cuya construcción prototípica se realiza mediante sintagmas nominales (muchas veces crípticos respecto al tema que tratan, con función más apelativa que informativa):

[Ej. 12] Sin tregua [Editorial EP, 10.01.2011]

Los niveles de narratividad son graduables según diferentes factores sintácticos e informativos; así, existen titulares con clara vocación de relato, mientras otros solo exponen hechos. Para atender a estos niveles analizamos el número de categorías narrativas incluidas en el texto, el tiempo verbal y la semántica del verbo. El titular prototípico es un sintagma oracional que aporta información sobre los dos actantes principales (sujeto y objeto) y un circunstante, que a su vez puede ser nominal o proposicional (sobre todo, subordinadas finales):

[Ej. 13] Unió Mallorquina pagaba a los jefes gitanos con billetes de $500 €$ en un bar [EM, 19.02.2011]

[Ej. 14] Gadafi arma a sus seguidores para defender Trípoli a sangre y fuego [EP, 27.02.2011]

[Ej. 15] Zapatero presiona a los sindicatos y a la patronal para que asuman su pacto con Merkel [EM, 15.03.2011]

En menor proporción (16,8\%) encontramos titulares que aun siendo narrativos, no tienen sintaxis oracional sino nominal, o con estructuras de estilo directo:

\footnotetext{
${ }^{12}$ Se suma a esta dimensión narrativa la idea de que el encuadre político es, además, un proceso diacrónico (Entman 2010: 401), sometido a la temporalidad que jalonan las citas electorales.
} 
[Ej. 16] Dos militares mutilados en un atentado en Afganistán [EM, 19.06.2011]

[Ej. 17] “Aprecio mucho al Bigotes, es cariñoso” [EP, 27.06.2011]

[Ej. 18] Feijóo: "Yo no me reuní en una gasolinera para hablar de subvenciones” [EM, 09.10.2011]

Por lo que se refiere al tiempo verbal, aunque los verbos en pasado son más prototípicamente narrativos que los de presente o futuro, los titulares proposicionales recurren mayoritariamente $(84,5 \%)$ a verbos en presente, algo que se relaciona también con el tipo de texto que constituye la noticia. En cuanto al tipo de verbo, puede haber titulares con riqueza categorial pero cuyo verbo es más procesual que agentivo, lo que supone que el nivel de narratividad sea menor:

[Ej. 19] La crisis agranda la fractura política en una debilitada UE [EP. 25.10.2011]

Por último, el nivel mínimo de narratividad corresponde a titulares descriptivos que suelen acompañar a una imagen, o al discurso referido. En este último caso, la noticia es un acto de habla que se carga de valor informativo por su sujeto de enunciación:

[Ej. 20] La CEOE desmiente a Zapatero y ve lejano el pacto sobre convenio [EM, 26.05.2011]

Obviamente, que alguien diga algo no constituye exactamente un relato, pero el acto de habla activa el marco narrativo en que tiene lugar, y que previsiblemente se desarrolla en el texto de la noticia.

\subsection{La estrategia de encuadre en el nivel interactivo}

En este punto nos referimos a la intertextualidad de los titulares, es decir, al modo en que estos se hacen eco no ya de los hechos políticos, sino del discurso político perteneciente a los actores políticos institucionales. Recurrir a la estrategia de encuadre intertextual supone inhibir la propia voz para dejar hablar al protagonista de la noticia, una inhibición que correlaciona con la objetividad periodística.

Gran parte de los titulares analizados refieren noticias que surgen a partir de actos de habla y/o acontecimientos comunicativos (a veces llamados (Hurtado 2009: 192) “titulares declarativos”); el discurso referido cobra, así, un protagonismo notable, superior al del discurso político original. En este sentido el discurso periodístico, como discurso político, es en gran medida un discurso ecoico, dependiente de una enunciación política previa. Un 29\% de los titulares recogen explícitamente un acto de habla 
protagonizado por actores políticos, de los cuales un 62,2\% son discurso referido en estilo indirecto, y un 37,8\% reproducen en estilo directo el mensaje de algún personaje político (la reproducción puede ser completa o parcial).

En el discurso de los representantes políticos la cita cumple dos funciones básicas: activar el contraste partidista cuando se citan palabras del oponente político, o recurrir al argumento de autoridad cuando se citan palabras de un representante del propio partido; supone, por tanto, un mecanismo subsidiario de la estrategia intencional de ataque o alabanza. Sin embargo, comprobamos que en la enunciación incrustada que supone el periodismo estas funciones se difuminan, aunque pueden mantenerse implícitamente, y la función fundamental del discurso referido es referencial, informativa.

\section{Conclusiones}

En este trabajo hemos revisado la aplicación de varias estrategias lingüísticas de encuadre en un corpus de titulares de noticias de portada de El País y El Mundo; hemos relacionado este uso con el que aparece en el discurso de los partidos políticos y con las investigaciones que atribuyen a la prensa española una cierta adscripción partidista. En el análisis de las estrategias de encuadre vinculadas al emisor comprobamos que los titulares de las noticias de prensa no muestran un uso coincidente con el que se aprecia en el discurso de la comunicación política de partido. Así, la estrategia intencional muestra una mayoría de actos de habla representativos (frente al predominio expresivo del discurso de los partidos y sus representantes), y la estrategia léxica no explota la dimensión prevaricadora de la designación, como ocurre en el uso político.

En el ámbito textual, encontramos correspondencia entre la dimensión narrativa del discurso político y la naturaleza igualmente narrativa de la noticia periodística que encabezan los titulares analizados; frente a otros géneros como el editorial o la columna, predominan los titulares informativos y proposicionales, sin que haya claras muestras de superestructura argumentativa. Por último, la dimensión interactiva del encuadre se aleja también del uso de los partidos políticos, de manera que el discurso referido (directo o indirecto) no se pone al servicio de la intención expresiva, sino representativa. En definitiva, el salto enunciativo que supone trasladar la comunicación política (oficial, institucional) al periodismo político (excluyendo, claro está, los géneros de opinión), 
impone una transformación discursiva que obliga a modificar las estrategias lingüísticas de encuadre, incluso en los casos en que los marcos interpretativos no resultan cuestionados. Tanto la intencionalidad básica representativa del periodismo como la propia textura narrativa de la noticia, explican las diferencias con la enunciación de los partidos políticos. Esta diferencia en el uso discursivo permite afirmar que el posible alineamiento partidista de las noticias del corpus analizado no emerge en aspectos implícitos del encuadre, como la ilocutividad expresiva, la designación prevaricadora o la estructura argumentativa, sino que debe buscarse en elementos explícitos de los textos, como el sesgo informativo en los contenidos, la selección de los temas de actualidad, o la dosificación informativa en cada noticia.

Bibliografía

Berganza, M. Rosa; Van Dalen, Arjen \& Chaparro, M. Ángeles (2010): “La percepción mutua de las relaciones entre periodistas parlamentarios y miembros del Congreso y de su influencia en las agendas política y mediática”, Revista de Comunicación 9, pp. 7-25. http://rcom.medianewsonline.com/pdf/2010/Art007-25.pdf

Bruner, Jerome Seymur (1986): Realidad mental y mundos posibles. Los actos de la imaginación que dan sentido a la experiencia, Barcelona, Gedisa, 1996. Trad. de Beatriz López.

Capella, Joseph N. y Jamieson, Kathleen Hall (1996): “News Frames, Political Cynicism and Media Cynicism”, Annals of the American Academy of Political and Social Science, July 1996, 71-84.

Casero Ripollés, Andreu (2008): “Modelos de relación entre periodistas y políticos: la perspectiva de la negociación constante”, Estudios sobre el mensaje periodístico, 14: $111-128$.

Casero Ripollés, Andreu \& López Rabadán, Pablo (2012): “La evolución de las fuentes informativas en el periodismo español”, III Congreso Internacional “Comunicación y Riesgo”. Universitat Rovira i Virgili. Tarragona, 18-20 enero 2012. http://www.aeic2012tarragona.org/comunicacions_cd/ok/311.pdf 
Castells Olivan, Manuel (2009): Comunicación y poder, Madrid, Alianza Editorial. Trad. de María Hernández.

Edelman, Murray (1985): “Political Language and Political Reality”, Policy Studies, 18, 10-19.

Entman, Robert M. (1991): "Framing US coverage of international news: contrast in narratives of the KAL and Iran air incidents”, Journal of Communication 41, 6-27.

Entman, Robert M. (1993): “Framing: Towards Clarification of a Fractured Paradigm”, Journal of Communication 43 (4), 51-58.

Entman, Robert M. (2009): Projections of power: Framing news, public opinion, and US foreign policy, University of Chicago Press.

Entman, Robert M. (2010): "Media framing biases and political power: Explaining slant in news of Campaign 2008”, Journalism 11.4: 389-408.

Fillmore, Charles (1976): "Frame semantics and the nature of language”, Annals of the New York Academy Sciences 280, 20-32.

Fisher, Walter R. (1984): "Narration as a Human Communication Paradigm: The Case of Public Moral Argument”, Communication Monographs 51, 1-22.

Fontcuberta, Mar de (1996): La noticia. Pistas para percibir el mundo, Barcelona: Bosch Comunicación.

Gallardo-Paúls, Beatriz (2013): “Niveles pragmáticos y cognición: estrategias discursivas de encuadre en el discurso político”, Anthropos 239, 191-210.

Gallardo-Paúls, Beatriz (2014): Usos políticos del lenguaje. Un discurso paradójico, Barcelona: Anthropos.

Hanitzsch, Thomas et al. (2011): “Mapping journalism cultures across nations”, Journalism Studies, 12: 3, 273-293. DOI: 10.1080/1461670X.2010.512502

Hopmann, David Nicolas; Van Aelst, Peter \& Legnante, Guido (2011): "Political balance in the news: A review of concepts, operationalizations and key findings”, Journalism 13(2), 240-257. DOI: 10.1177/1464884911427804.

Howarth, David (2005): “Aplicando la teoría del discurso: el método de la articulación”, Studia Politicae 5, 37-88. 
Hurtado González, Silvia (2009): "Algunas peculiaridades de los titulares de actos de habla en la prensa española e hispanoamericana”, Zer: Revista de estudios de comunicación 27 (2009): 189-202.

Jorques, Daniel (2000): Discurso e información. Estructura de la prensa escrita, Cádiz: Universidad de Cádiz.

Kalb, Marvin (1992): “Press-Politics and Improving the Public Dialogue”, Political Communication Report 3: 1-3.

Krippendorff, Klaus (1990): Metodología de análisis de contenido. Teoría y Práctica, Barcelona: Paidós.

Lakoff, George (1992): “Metaphors and the War: The Metaphor system Used to Justify War in the Gulf”, en M. Pütz (Ed.) (1992): Thirty Years of Linguistic Evolution: Studies in Honor of René Dirven on the Occasion of His Sixtieth Birthday, Philadelphia: John Benjamins, 463-481.

Lakoff, George (2004): No pienses en un elefante, Madrid: UCM, 2007. Trad. de Magdalena Mora.

Mazzoleni, Gianpietro (1998): La comunicación política, Madrid: Alianza, 2010. Trad. de Josefa Linares de la Puerta.

McNair, Brian (2000): Journalism and Democracy. Evaluation of Political Public Sphere, London: Routledge.

Núñez Ladevéze, Luis (1991): Manual para periodismo. Veinte lecciones sobre el contexto, el lenguaje y el texto de la información, Madrid: Pirámide.

Ricoeur, Paul (1987): “La fragilidad del lenguaje político”, Signo y pensamiento 15, 33-43.

Sánchez Aranda, José Javier y Berganza Conde, M. Rosa (1999): “Framing en la prensa española: la información sobre la muerte y funeral de Diana de Gales”, Comunicación y Sociedad XII/1, 137-161.

Scheufele, Dietram A. (1999): "Framing as a theory of media effects", Journal of communication 49.1: 103-122. 
Thogmartin, Clyde (1991) "The pragmatics of french newspaper headlines", en J. Verschueren (ed.): Levels of linguistic adaptation, Amsterdam-Philadelphia: John Benjamins, 249-266.

Van Dalen, Arjen (2010): Political journalism in comparative perspective. Summary. http://static.sdu.dk/mediafiles//6/2/3/\%7B623BAE19-C202-4F9F-9A4FEEEAA01F535E\%7DArjenvanDalen2010_PoliticalArjenVanDalenJournalisminComparativePerspective_Summary.pdf

Van Dalen, Arjen; Albaek, Erik \& de Vreese, Claes (2012): “Different roles, different content? A four-country comparison of the role conceptions and reporting style of political journalists”, Journalism 13 (7), 903-922. DOI: 10.1177/ 1464884911431538

Van Dijk, Teun Adrianus (1980): La noticia como discurso, Barcelona: Paidós, 1990. Trad. Guillermo Gal.

Van Zoonen, Liesbet (2005): Entertaining the citizen: When politics and popular culture converge, Oxford: Rowman \& Littlefield.

Wodak, Ruth (1989): “1968: The power of political jargon -a ‘Club 2' discussion”, en R. Wodak (Ed.): Language, Power and Ideology, Amsterdam: John Benjamins, 137-163.

Recibido: 19 enero 2014

Aceptado: 7 abril 2014

Revisado: 14 mayo 2014

Publicado: 31 mayo 2014

Actualizado: 2 junio 2014 